\title{
Characterization of the Minimum Detectable Fault of Interval Observers by using Set-invariance Theory
}

\author{
Masoud Pourasghar ${ }^{1,2}$, Vicenç Puig ${ }^{1,2}$ and Carlos Ocampo-Martinez ${ }^{1,2}$
}

\begin{abstract}
This paper addresses the characterization of the minimum detectable fault when using interval observers. The interval observers consider both input and uncertainty as unknown but bounded. The minimum detectable fault using interval observers is characterized by means of residual sensitivity and invariant sets. The design of such observers is performed by using zonotopic-set representations. The mathematical expression of the minimum magnitude of the fault that can be detected is derived for a given type of faults in separate formulations. Finally, a simulation example is employed to illustrate and discuss the effectiveness of the obtained results.
\end{abstract}

\section{INTRODUCTION}

The demand of increasing safety and reliability of the modern industrial control systems has attracted much attention from many scientific communities to explore about the reason of losing the system performance. Occurrence of the fault affects the system behaviour preventing to perform in the expected way. Therefore, detecting and isolating the fault and preparing the system for facing this event are important research issues over the past two decade. Fault Detection and Isolation (FDI) and Fault-Tolerant Control (FTC) schemes are responsible to lead the system to reach the expected objectives, see e.g., [4], [5] and [7].

Alternatively, dealing with estimating the fault is another hot topic in fault detection context [3], [19]. Due to unexpected existence of perturbations, disturbances and noises, estimating the fault value is quite difficult in a faulty system by using current FDI approaches [1], [8]. Therefore, the estimation of the magnitude of the fault, known as fault estimation, and its related approaches has been started to be researched, see e.g., [12], [17], [8] and the references therein. Moreover, having the accurate fault information can increase designing accuracy of the controller with FTC techniques [18].

Based on the scientific literature, there are various approaches in fault estimation area [13] and [11], but the most common one is the observer-based approach [14]. This approach is fundamentally based on designing the proper output observer that allows generating the residual signal and comparing the residual evaluation with pre-defined threshold (zero in ideal case). It must be guaranteed that the residual does not exceed the threshold, otherwise, a fault alarm is generated [15].

\footnotetext{
${ }^{1}$ Supervision, Safety and Automatic Control Research Center (CS2AC) of the Universitat Politècnica de Catalunya, Campus de Terrassa, Gaia Building, Rambla Sant Nebridi, 22, 08222 Terrassa, Barcelona, Spain.

${ }^{2}$ Automatic Control Department of the Universitat Politècnica de Catalunya, Institut de Robòtica i Informàtica Industrial (CSICUPC). Llorens i Artigas, 4-6, 08028 Barcelona, Spain. E-mail: \{ mpourasgharlaf, vpuig, cocampo\}eiri.upc.edu
}

On the other hand, set invariance is another FDI method that can be used in this context [10], [15], [16]. This approach obtains the residual set in the healthy mode considering unknown but bounded uncertainties. This invariant set can be used for showing the healthy mode. Therefore, if the residual in faulty mode is outside this invariant set means that a fault has occurred in the system [16].

Moreover, the accuracy of the fault detection is highly affected by the modeling uncertainties. The characterization of the minimum detectable fault is important in order to know the limits of performance of the considered FDI scheme. When using interval observers, the minimum detectable fault is highly dependent on the observer gain as discussed in [9].

The main contribution of this paper is twofold. First, the characterization of minimum detectable faults in case of using interval observers will be carried out using set-invariant theory in the case of single input/single output systems. Second, the minimum detectable fault is derived by means of mathematical expressions for each type of fault (input sensor fault, output sensor fault and actuator fault), separately. The characterization of the invariant sets and their computations will be done using zonotopes.

Regarding the structure of the paper, Section II introduces fault detection using interval observers and set-invariance theory. The characterization of the minimum detectable fault is done in Section III. An example based on a two-tanks system is used in order to illustrate the results in Section IV. Finally, the general conclusion are drawn in Section V.

\section{Fault Detection Using Interval Observers}

\section{A. Problem set-up}

In this paper, the study of the fault detection methods based on set-theoretical approach is focused on linear discrete time uncertain dynamical model as

$$
\begin{aligned}
x_{k+1} & =A x_{k}+B u_{k}+\omega_{k}, \\
y_{k} & =C x_{k}+v_{k},
\end{aligned}
$$

where

- $u \in \mathbb{R}^{p}, y \in \mathbb{R}^{q}$ and $x \in \mathbb{R}^{n}$ are the input, output and the state vectors, respectively.

- $A \in \mathbb{R}^{n \times n}, B \in \mathbb{R}^{n \times p}$ and $C \in \mathbb{R}^{q \times n}$ are the state-space matrices.

- $\omega \in \mathbb{R}^{r}$ and $v \in \mathbb{R}^{q}$ are measurement disturbance and process noise, respectively.

- $k \in \mathbb{Z}$ indicates the $k$-th discrete-time instant.

Moreover, the input vector, the measurement disturbance and the process noise are considered unknown but bounded, i.e., 
$u_{k} \in U, \omega_{k} \in W$ and $v_{k} \in V$, where $U, W$ and $V$ are interval boxes defined as follows:

$$
\begin{aligned}
U & =\left\{u_{k} \in \mathbb{R}^{p}: \underline{u}_{k}<u_{k}<\bar{u}_{k}\right\}, \\
W & =\left\{\omega_{k} \in \mathbb{R}^{r}: \underline{\omega}_{k}<\omega_{k}<\bar{\omega}_{k}\right\}, \\
V & =\left\{v_{k} \in \mathbb{R}^{q}: \underline{v}_{k}<v_{k}<\bar{v}_{k}\right\},
\end{aligned}
$$

where notations $\bar{u}, \bar{\omega}$ and $\bar{v}$ are the maximum values of $u_{k}$, $\omega_{k}$ and $v$, respectively. Moreover, $\underline{u}, \underline{\omega}$ and $\underline{v}$ are used for indicating the minimum values of $u, \omega$ and $v$ of each interval boxes, respectively.

Also, sets $U, W$ and $V$ can be re-written with zonotopic center-segments form as

$$
\begin{aligned}
U & =u^{c} \oplus H_{\bar{u}} \mathbb{B}^{p}, \\
W & =\omega^{c} \oplus H_{\bar{\omega}} \mathbb{B}^{r}, \\
V & =v^{c} \oplus H_{\bar{v}} \mathbb{B}^{q},
\end{aligned}
$$

where $u^{c}, \omega^{c}$ and $v^{c}$ denote the centers of the input, the measurement disturbance and the process noise zonotopes with their generator matrices $H_{\bar{u}} \in \mathbb{R}^{p \times p}, H_{\bar{\omega}} \in \mathbb{R}^{r \times r}$ and $H_{\bar{v}} \in \mathbb{R}^{q \times q}$, respectively.

\section{B. Interval-observer-based fault detection}

Based on the dynamical model (1), the mathematical formulation of the interval observer is expressed as

$$
\begin{aligned}
\hat{X}_{k+1} & =(A-K C) \hat{X}_{k} \oplus B U \oplus\left\{K y_{k}\right\} \oplus(-K) V \oplus W, \\
\hat{Y}_{k} & =C \hat{X}_{k} \oplus V,
\end{aligned}
$$

where $\hat{X}$ and $\hat{Y}$ denote the predicted zonotopic set for the state and output, respectively. Furthermore, $K$ denotes the observer gain.

Considering (3), the observer can be re-written as a zonotope. Thus, the centers and the segments of this predicted zonotope are propagated as

$$
\begin{aligned}
\hat{x}_{k+1}^{c} & =A_{o b s} \hat{x}_{k}^{c}+B u_{k}^{c}+K y_{k}-K v^{c}+\omega^{c}, \\
\hat{y}_{k}^{c} & =C \hat{x}_{k}^{c}+v^{c}, \\
\hat{H}_{k+1}^{x} & =\left[\begin{array}{llll}
A_{o b s} \hat{H}_{k}^{x} & B H_{\bar{u}} & -K H_{\bar{v}} & H_{\bar{\omega}}
\end{array}\right], \\
\hat{H}_{k}^{y} & =\left[\begin{array}{lll}
C \hat{H}_{k}^{x} & H_{\bar{v}}
\end{array}\right],
\end{aligned}
$$

where

- $A_{o b s}=(A-K C)$.

- $\hat{x}_{k+1}^{c}$ and $\hat{H}_{k+1}^{x}$ : the center and the segments of the state predicted zonotopic set, respectively.

- $\hat{y}_{k}^{c}$ and $\hat{H}_{k+1}^{y}$ : the center and the segments of the output predicted zonotopic set, respectively.

Therefore, the zonotopic residual $R_{k}$ can be computed for this zonotopic observer at each time instant as

$$
R_{k}=r_{k}^{c} \oplus H_{k}^{r} \mathbb{B}^{q},
$$

where $r_{k}^{c}$ and $H_{k}^{r}$ denote the center and the segments of the residual zonotope, respectively. Additionally, these center and segments are derived as

$$
\begin{aligned}
r_{k}^{c} & =y_{k}-\hat{y}_{k}^{c} \\
& =\left(C x_{k}+v_{k}\right)-\left(C \hat{x}_{k}^{c}+v^{c}\right) \\
& =C\left(x_{k}-\hat{x}_{k}^{c}\right)+v_{k}-v^{c},
\end{aligned}
$$

and

$$
H_{k}^{r}=\hat{H}_{k}^{y}
$$

A fault detection test with interval observers is based on generating the residual signal by comparing the measurement $y_{k}$ in (1) with its prediction in (4) as

$$
R_{k}=\left\{y_{k}\right\} \oplus\left(-\hat{Y}_{k}\right),
$$

where $R$ is the residual set is usually bounded by it interval hull $\square R$ for fault detection purposes. Therefore, the fault detection test is done by checking the consistency of $0 \in$ $\square R_{k}$, thus the existence of fault will be detected if $0 \notin \square R_{k}$.

\section{Set-invariance-based fault detection}

The set-invariance-based method can also be used for detecting the fault in the system by means of the interval observer in (4).

Definition 2.1 (RPI Set): Consider the dynamical model $x_{k+1}=f\left(x_{k}, \omega_{k}\right)$, where $x_{k} \in X$ and $\omega_{k} \in W$. A set $X$ is a RPI set if for $X$ and $W$, it holds that $x_{k+1} \in X$.

Based on Definition 2.1, the RPI set $\breve{R}_{k}$ with the center $\breve{r}_{k}^{c}$ and segments $\breve{H}_{k}^{r}$ for the obtained residual in (6) is written as

$$
\check{R}_{k}=\check{r}_{k}^{c} \oplus \check{H}_{k}^{r} \mathbb{B}^{q} .
$$

Hence, whenever $R_{k}$ goes into its RPI set, it will always remain inside of the RPI set.

Furthermore, the fault detection test with the invariant-setbased approach is carried out by comparing the residual set $R_{k}$, which is computed on-line for each time instant, with the RPI residual set for healthy mode $\check{R}_{k}$, which is obtained off-line. The fault is detected if $R_{k} \cap \check{R}_{k}=\emptyset$, otherwise, the system is still in healthy mode.

\section{Minimum Detectable Fault Characterization}

In this section, the minimum magnitude of fault that can be detected is analyzed from the theoretical point of view. This analysis is done following two approaches:

- Residual sensitivity analysis

- Set-invariance theory

\section{A. Minimum detectable fault using residual sensitivity anal- ysis}

The fault can be classified into actuator fault, input sensor fault and output sensor fault based on the location of its occurrence in the system. The dynamical model (1) can be re-written by considering all types of fault as

$$
\begin{aligned}
x_{k+1} & =A x_{k}+B\left(u_{k}+F_{u} f_{u}\right)+\omega_{k}+F_{a} f_{a}, \\
y_{k} & =C x_{k}+v_{k}+F_{y} f_{y},
\end{aligned}
$$

where $f_{y}, f_{u}$ and $f_{a}$ denote the output sensor fault, the input sensor fault and the actuator fault, respectively, with their associated matrices $F_{y}, F_{u}$ and $F_{a}$ of suitable dimensions.

Assumption 3.1: The fault is considered unknown but bounded. 
Definition 3.1 (Residual Sensitivity): The analytical expression of this concept is defined as

$$
S_{f}=\frac{\partial r}{\partial f}
$$

where $S_{f}$ shows the sensitivity of the residual and indicates the effect of the given fault $f$ on the residual $r$.

Definition 3.2 (Minimum Detectable fault): The minimum detectable fault is the magnitude of the fault that can bring the residual set out of its RPI set. Moreover, the residual in faulty situation can be written as

$$
R_{k}^{f}=\stackrel{\circ}{R}_{k}+\left(S_{f} f\right),
$$

where $R_{k}^{f}$ indicate the residual set in faulty situation and $\stackrel{\circ}{R}_{k}$ shows the nominal residual set that can be computed by considering the system healthy mode as

$$
\begin{aligned}
\stackrel{\circ}{R}_{k} & =y_{k}-\hat{Y}_{k} \\
& =\left\{C x_{k}+v_{k}\right\} \oplus\left\{-\left(C \hat{X}_{k}^{c}\right) \oplus(V)\right\} \\
& =C\left\{\left\{x_{k}\right\}-\left(\hat{X}_{k}\right)\right\} \oplus\left\{v_{k}\right\} \oplus(-V) .
\end{aligned}
$$

Therefore, $R_{k}^{f} \notin \check{R}_{k}$ if the system is faulty. In other words, (13) is written as

$$
\stackrel{\circ}{R}_{k}+\left(S_{f} f_{\text {min }}\right) \notin \check{R}_{k}
$$

Consequently, the minimum detectable fault based on its residual sensitivity can be computed as

$$
\begin{aligned}
& f_{\text {min }}>S_{f}^{\dagger}{\stackrel{\max }{\operatorname{Rax}_{k}},}^{\bullet} \\
& f_{\text {min }}<S_{f}^{\dagger}{\stackrel{\bullet}{R_{k}}}_{\text {min }},
\end{aligned}
$$

where the notation $(\cdot)^{\dagger}$ is used for indicating the MoorePenrose pseudo-inverse matrix. Also, $\dot{R}_{k}$ and $\dot{R}_{k}$ max minimum and the maximum values of discrepancy of the invariant zonotope and the nominal residual $\left(\dot{R}_{k}=\check{R}_{k}-\stackrel{\circ}{R}_{k}\right)$, where $\stackrel{\circ}{R}_{k}$ is the nominal residual that contains the effect of the model parameter uncertainties.

1) Minimum detectable output sensor fault: The dynamical model that is considered for dealing with this type of fault can be written as

$$
\begin{aligned}
x_{k+1} & =A x_{k}+B u_{k}+\omega_{k}, \\
y_{k} & =C x_{k}+v_{k}+F_{y} f_{y} .
\end{aligned}
$$

According to (12), the sensitivity of the residual to a given fault is needed. Thus, the residual affected by the output sensor fault can be obtained as

$$
\begin{aligned}
r_{k}^{y} & =y_{k}-\hat{y}_{k} \\
& =\left(C x_{k}+v_{k}+F_{y} f_{y}\right)-\left(C \hat{x}_{k}-V\right),
\end{aligned}
$$

where $r_{k}^{y}$ denotes the residual of output sensor fault. Additionally, the shift operator $q$ is used to show the influence of the fault over $\hat{x}_{k}$ as

$$
\hat{x}_{k}=\Phi\left(B U+K y_{k}-K V+W\right),
$$

where

$$
\begin{aligned}
& \Phi=(q I-\Gamma)^{\dagger}, \\
& \Gamma=A-K C .
\end{aligned}
$$

Now, the residual can be obtained by substituting (18) into (17). Moreover, the sensitivity of the residual to the output sensor fault based on (12) is expressed as $S_{f_{y}}=\frac{\partial r_{k}^{y}}{\partial f_{y}}$, where $S_{f_{y}}$ denotes the sensitivity of the residual to the considered fault. Also, $S_{f_{y}}$ can be expressed as follows:

$$
S_{f_{y}}=F_{y}(I-C \Phi K) .
$$

Therefore, based on the inequalities in (15), the minimum detectable output sensor fault $f_{\text {min }}^{y}$ can be derived as

$$
\begin{gathered}
f_{\text {min }}^{y}>\left(F_{y}(I-C \Phi K)\right)^{\dagger} \stackrel{\dot{R}_{k},}{\max _{k},} \\
f_{\text {min }}^{y}<\left(F_{y}(I-C \Phi K)\right)^{\dagger} \stackrel{\dot{R}_{k} .}{\min _{\text {min }}}
\end{gathered}
$$

2) Minimum detectable input sensor fault: The faulty dynamical model for input sensor fault is considered as

$$
\begin{aligned}
x_{k+1} & =A x_{k}+B\left(u_{k}+F_{u} f_{u}\right)+\omega_{k}, \\
y_{k} & =C x_{k}+v_{k} .
\end{aligned}
$$

Also, the same procedure is used for finding the minimum detectable input sensor fault. First, the residual is obtained as

$$
\begin{aligned}
r_{k}^{u} & =y_{k}-\hat{y}_{k} \\
& =\left(C x_{k}+v_{k}\right)-\left(C \hat{x}_{k}-V\right),
\end{aligned}
$$

where $r_{k}^{u}$ denotes the residual when the fault has occurred in the input of the sensor. In addition, $\hat{x}_{k}$ is derived as in (18).

At the end, according to (22) and (18), by considering $S_{f_{u}}=\frac{\partial r_{k}^{u}}{\partial f_{u}}$, the residual sensitivity to the input sensor fault can be obtained as

$$
S_{f_{u}}=-F_{u}(C \Phi B) .
$$

Thus, the inequalities in (15) are re-written for finding the minimum detectable output sensor fault $f_{\min }^{u}$ as

$$
\begin{gathered}
f_{\text {min }}^{u}>\left(-F_{u}(C \Phi B)\right)^{\dagger} \begin{array}{c}
\stackrel{R}{R}_{k}, \\
\max
\end{array} \\
f_{\text {min }}^{u}<\left(-F_{u}(C \Phi B)\right)^{\dagger} \stackrel{\dot{R}_{k}}{\dot{R}_{\min }}
\end{gathered}
$$

3) Minimum detectable actuator fault: The considered faulty dynamical model in this subsection is as follows:

$$
\begin{aligned}
x_{k+1} & =A x_{k}+B u_{k}+\omega_{k}+F_{a} f_{a}, \\
y_{k} & =C x_{k}+v_{k} .
\end{aligned}
$$

Same as two previous subsections, the residual of the system affected by actuator fault $r_{k}^{a}$, is obtained as

$$
\begin{aligned}
r_{k}^{a} & =y_{k}-\hat{y}_{k} \\
& =\left(C x_{k}+v_{k}\right)-\left(C \hat{x}_{k}-V\right) .
\end{aligned}
$$


Furthermore, the residual sensitivity to a system with actuator fault by considering (18) in order to show the effect of fault on $\hat{x}_{k}$ can be computed as

$$
S_{f_{a}}=\frac{\partial r_{k}^{a}}{\partial f_{a}}=F_{a}(I-C \Phi K) C \Lambda
$$

where $\Lambda=(q I-A)^{\dagger}$.

At the end, the minimum detectable actuator fault $f_{\text {min }}^{a}$ can be obtained by substituting the (27) into (15) as

$$
\begin{aligned}
& f_{\text {min }}^{a}>\left(F_{a}(I-C \Phi K) C \Lambda\right)^{\dagger}{\stackrel{\max }{R_{k}},}^{\bullet}, \\
& f_{\text {min }}^{a}<\left(F_{a}(I-C \Phi K) C \Lambda\right)^{\dagger} \underset{\text { min }}{\stackrel{\bullet}{R_{k}}} .
\end{aligned}
$$

B. Minimum detectable fault based on interval observers by using set-invariance theory

Alternatively, the minimum detectable fault can be explored based on the interval observer by using set-invariance theory. This method is based on the comparison of the interval hull of faulty zonotope and invariant zonotopes. This zonotope with the center $r_{k}^{c, f}$ and the segments $H_{k}^{r, f}$ are expressed as

$$
R_{k}^{f}=r_{k}^{c, f} \oplus H_{k}^{r, f} \mathbb{B}^{q}
$$

Therefore, the maximum $Q_{\max }$ and the minimum $Q_{\min }$ bounds of the smallest box that contains this zonotope is computed by using the interval hull of the zonotope in (29) as

$$
\begin{aligned}
Q_{\text {max }} & =r_{k}^{c, f}+\left\|H_{k}^{r, f}\right\|_{1}, \\
Q_{\text {min }} & =r_{k}^{c, f}-\left\|H_{k}^{r, f}\right\|_{1},
\end{aligned}
$$

where $\|$.$\| denotes the 1-norm of the segments matrix.$

On the other hand, the maximum and minimum bounds for the interval hull of the invariant zonotope in (10) are derived as

$$
\begin{gathered}
\check{Q}_{\text {max }}=\check{r}_{k}^{c}+\left\|\check{H}_{k}^{r}\right\|_{1}, \\
\check{Q}_{\text {min }}=\check{r}_{k}^{c}-\left\|\check{H}_{k}^{r}\right\|_{1},
\end{gathered}
$$

where $\check{Q}_{\max }$ and $\check{Q}_{\min }$ are used for characterizing the maximum and minimum bounds, respectively. Also, the center $\check{r}_{k}^{c}$ and the segments $\breve{H}_{k}^{r}$ are obtained as

$$
\begin{aligned}
\check{r}_{k}^{c} & =C\left(x_{k}-\hat{x}_{k}^{c}\right)+\left(v_{k}-v^{c}\right), \\
\check{H}_{k}^{r} & =\left[\begin{array}{ll}
C \hat{H}_{k}^{x} & -H_{\bar{v}}
\end{array}\right] .
\end{aligned}
$$

In addition, in faulty situation, there is not intersection between $R_{k}^{f}$ and $\check{R}_{k}$, hence, the following inequalities can be obtained in faulty mode:

$$
\begin{aligned}
& Q_{\text {min }}>\check{Q}_{\text {max }}, \\
& Q_{\text {max }}<\check{Q}_{\text {min }} .
\end{aligned}
$$

Therefore, the following conditions should be satisfied in the faulty system:

$$
\begin{aligned}
& r_{k}^{c, f}-\check{r}_{k}^{c}>\left\|H_{k}^{r, f}\right\|_{1}+\left\|\check{H}_{k}^{r}\right\|_{1}, \\
& r_{k}^{c, f}-\check{r}_{k}^{c}<-\left(\left\|H_{k}^{r, f}\right\|_{1}+\left\|\check{H}_{k}^{r}\right\|_{1}\right) .
\end{aligned}
$$

Using the method described above, the difference between the sets in faulty and healthy modes generates the conditions in (33). Based on these conditions, the minimum detectable fault can be computed by using the centers and the segments of the invariant zonotope and the zonotope in faulty mode at each time step.

1) Minimum detectable output sensor fault: By considering the main concept that is mentioned above, the residual zonotope $R_{k}^{f_{y}}$ is obtained for the system with output sensor fault based on dynamical model in (16) as

$$
R_{k}^{f_{y}}=r_{k}^{c, f_{y}} \oplus H_{k}^{r, f_{y}} \mathbb{B}^{q}
$$

where $r_{k}^{c, f_{y}}$ and $H_{k}^{r, f_{y}}$ denote the center and the segments of the residual zonotope, respectively.

Hence, according to the observer in (5) and also the residual in (17), the center and segments can be obtained as

$$
\begin{aligned}
r_{k}^{c, f_{y}} & =y_{k}-\left(C \hat{x}_{k}^{c, f_{y}}+v^{c}\right), \\
H_{k}^{r, f_{y}} & =\left[\begin{array}{lll}
C \hat{H}_{k}^{x, f_{y}} & F_{y} H_{\overline{f_{y}}} & -H_{\bar{v}}
\end{array}\right],
\end{aligned}
$$

where

$$
\begin{aligned}
\hat{x}_{k}^{c, f_{y}} & =\Phi\left(B u^{c}+K y_{k}-K v^{c}+\omega^{c}\right), \\
\hat{H}_{k}^{x, f_{y}} & =\Phi\left[\begin{array}{llll}
B H_{\bar{u}} & K F_{y} H_{\overline{f_{y}}} & -K H_{\bar{v}} & H_{\bar{\omega}}
\end{array}\right] .
\end{aligned}
$$

Therefore, the minimum detectable output sensor fault can be obtained by considering the residual zonotopes in (32) and (35) and also the inequalities in (33) as

$$
\begin{aligned}
& f_{\text {min }}^{y}>F_{y}^{\dagger} \cdot\left(C\left(\hat{x}_{k}^{c, f_{y}}-\hat{x}_{k}^{c}\right)+\left(\left\|\check{H}_{k}^{r}\right\|_{1}+\left\|H_{k}^{r, f_{y}}\right\|_{1}\right)\right), \\
& f_{\text {min }}^{y}<F_{y}^{\dagger} \cdot\left(C\left(\hat{x}_{k}^{c, f_{y}}-\hat{x}_{k}^{c}\right)-\left(\left\|\check{H}_{k}^{r}\right\|_{1}+\left\|H_{k}^{r, f_{y}}\right\|_{1}\right)\right) .
\end{aligned}
$$

2) Minimum detectable input sensor fault: The dynamical model in (21) is considered for the system with input sensor fault. The residual of this system is derived as

$$
R_{k}^{f_{u}}=r_{k}^{c, f_{u}} \oplus H_{k}^{r, f_{u}} \mathbb{B}^{q},
$$

where $R_{k}^{f_{u}}$ denotes the residual zonotope of the system for this case. Furthermore, its center $r_{k}^{c, f_{u}}$ and segments $H_{k}^{r, f_{u}}$ can be derived as

$$
\begin{aligned}
r_{k}^{c, f_{u}} & =y_{k}-\left(C \hat{x}_{k}^{c, f_{u}}+v^{c}\right), \\
H_{k}^{r, f_{u}} & =\left[\begin{array}{ll}
C \hat{H}_{k}^{x, f_{u}} & -H_{\bar{v}}
\end{array}\right],
\end{aligned}
$$

where

$$
\begin{aligned}
\hat{x}_{k}^{c, f_{u}} & =\Phi\left(B u^{c}+K y_{k}-K v^{c}+\omega^{c}\right), \\
\hat{H}_{k}^{x, f_{u}} & =\Phi\left[\begin{array}{llll}
B H_{\bar{u}} & K C \Lambda B F_{u} H_{\overline{f_{u}}} & -K H_{\bar{v}} & H_{\bar{\omega}}
\end{array}\right] .
\end{aligned}
$$

Hence, the minimum detectable input sensor fault can be obtained by using (32), (38) and (33) as

$$
\begin{aligned}
& f_{\text {min }}^{u}>\left(C \Lambda B F_{u}\right)^{\dagger} \cdot\left(C\left(\hat{x}_{k}^{c, f_{u}}-\hat{x}_{k}^{c}\right)+\left(\left\|\check{H}_{k}^{r}\right\|_{1}+\left\|H_{k}^{r, f_{u}}\right\|_{1}\right)\right), \\
& f_{\text {min }}^{u}<\left(C \Lambda B F_{u}\right)^{\dagger} \cdot\left(C\left(\hat{x}_{k}^{c, f_{u}}-\hat{x}_{k}^{c}\right)-\left(\left\|\check{H}_{k}^{r}\right\|_{1}+\left\|H_{k}^{r, f_{u}}\right\|_{1}\right)\right) .
\end{aligned}
$$


3) Minimum detectable actuator fault: The dynamical model (25) is considered for this case. The residual zonotope $R_{k}^{f_{a}}$ that is created by the system for facing this fault is derived as

$$
R_{k}^{f_{a}}=r_{k}^{c, f_{a}} \oplus H_{k}^{r, f_{a}} \mathbb{B}^{q} .
$$

Thus, the center $r_{k}^{c, f_{a}}$ and the segments $H_{k}^{r, f_{a}}$ of this zonotope are computed as

$$
\begin{aligned}
& r_{k}^{c, f_{a}}=y_{k}-\left(C \hat{x}_{k}^{c, f_{a}}+v^{c}\right), \\
& H_{k}^{r, f_{a}}=\left[\begin{array}{ll}
C \hat{H}_{k}^{x, f_{a}} & -H_{\bar{v}}
\end{array}\right],
\end{aligned}
$$

where

$$
\begin{aligned}
\hat{x}_{k}^{c, f_{a}} & =\Phi\left(B u^{c}+K y_{k}-K v^{c}+\omega^{c}\right), \\
\hat{H}_{k}^{x, f_{a}} & =\Phi\left[\begin{array}{llll}
B H_{\bar{u}} & K C \Lambda H_{\overline{f_{a}}} & -K H_{\bar{v}} & H_{\bar{\omega}}
\end{array}\right] .
\end{aligned}
$$

Therefore, according to the (32), (41) and (33) the minimum detactable fault is obtained as

$$
\begin{aligned}
& f_{\text {min }}^{a}>\left(C \Lambda F_{a}\right)^{\dagger} \cdot\left(C\left(\hat{x}_{k}^{c, f_{a}}-\hat{x}_{k}^{c}\right)+\left(\left\|\check{H}_{k}^{r}\right\|_{1}+\left\|H_{k}^{r, f_{a}}\right\|_{1}\right)\right), \\
& f_{\text {min }}^{a}<\left(C \Lambda F_{a}\right)^{\dagger} \cdot\left(C\left(\hat{x}_{k}^{c, f_{a}}-\hat{x}_{k}^{c}\right)-\left(\left\|\check{H}_{k}^{r}\right\|_{1}+\left\|H_{k}^{r, f_{a}}\right\|_{1}\right)\right) .
\end{aligned}
$$

\section{Comparative assessment}

This paper aims to obtain the characterization of the minimum detectable fault using sensitivity analysis and set invariance theory. From the mathematical point of view, the observer gain has an important influence on the minimum magnitude of the fault that can be detected with both approaches. The sensitivity analysis approach points out the influence of the observer gain on the time evolution of the residual sensitivity to a fault. On the other hand, the effect of the observer gain is shown on set invariance of the residual.

Additionally, a general comparison between the sensitivity analysis and the set invariance theory to compute the minimum detectable fault shows that the main difference between the presented approaches is based on their algorithms to generate the residual signal. With the sensitivity approach, the residual sets of both healthy and faulty modes are computed on-line. Then formulations in (20), (24) and (28) will be able to compute the minimum detectable fault. In contrast to the sensitivity analysis, the residual of the healthy mode is generated off-line in set invariance theory which leads to reduce the computational burden, when taking (36), (39) and (42) into account for calculating the minimum magnitude of the fault that can be detected. In other words, the computational complexity in invariant set-based approach does not play the crucial role in the approach since it uses an off-line method for predicting the state and the output sets. Therefore, the computational burden in sensitivity analysis is massively higher than in set invariance theory.

A detailed comparison between the considered approaches from the mathematical point of view can be done by comparing (20) with (36), (24) with (39) and (28) with (42) for minimum detectable output sensor fault, minimum detectable input sensor fault and minimum detectable actuator fault, respectively. It can be observed that computing the minimum detectable fault with both approaches is based on how the effect of fault is considered. In (20), (24) and (28) the effect of fault is modeled by means of fault sensitivity. Then, the minimum detectable fault is computed by comparing the set in healthy mode with the set in faulty mode which is obtained by multiplying the fault sensitivity into the healthy set. On the contrary, in (36), (39) and (42) the effect of fault is modeled on the center and the segments of the residual set, directly. Then, the minimum detectable fault is obtained by comparing the center and the segments of the residual set in healthy and faulty modes.

Furthermore, the main weakness of the set invariance approach for computing the magnitude of minimum detectable fault is related to the over-approximation (due to the effect of using interval hull of the zonotope instead of the zonotope for reducing the computational complexity) to generate the segments of the residual zonotope. As it can be seen from (36), (39) and (42), the interval hull of the residual zonotope in healthy and faulty modes are considered, while in (20), (24) and (28), this over-approximation is not considered because the residual sets in healthy and faulty modes are obtained by using zonotopes.

\section{Application Example}

\section{A. Description}

A two-tanks system is used to illustrate the minimum detectable fault analysis for interval observers using the approaches proposed in this paper. This two-tank system is a part of the well know four-tanks system benchmark proposed in [6]. Using the Euler discretization with a sampling time of $1 s$, the linearized model of this system can be written in the state-space form as in (1), where the state $x$ indicates the water level of the tanks. The parameters of the model are

- System matrices:

$$
\begin{aligned}
& -A=\left[\begin{array}{cc}
0.9984 & 0.04096 \\
0 & 0.959
\end{array}\right], B=\left[\begin{array}{c}
0.0002947 \\
0.01399
\end{array}\right], \\
& -E=\left[\begin{array}{c}
0.07173 \\
0
\end{array}\right], C=\left[\begin{array}{ll}
0.5 & 0
\end{array}\right] .
\end{aligned}
$$

- $\omega, v$ and $u$ denote the disturbance, the measurement noise and the input, respectively, which are considered unknown but bounded with the following offset magnitudes:

$$
\begin{aligned}
-H_{\omega} & =\left[\begin{array}{ll}
0.005 & 0.005
\end{array}\right]^{T}, \\
-H_{v} & =\left[\begin{array}{ll}
0.005 & 0.005
\end{array}\right]^{T}, \\
-H_{u} & =\left[\begin{array}{ll}
0.7 & 0.7
\end{array}\right]^{T} .
\end{aligned}
$$

- Observer gain $K$ has been design using Kalman filter approach according to [2].

- Initial conditions:

$$
\begin{aligned}
& -x_{0}=\left[\begin{array}{ll}
0 & 0
\end{array}\right]^{T}, \\
& -\hat{x}_{0}^{c}=\left[\begin{array}{ll}
0 & 0
\end{array}\right]^{T}, \hat{H}_{0}^{x}=\left[\begin{array}{cc}
0.01 & 0 \\
0 & 0.01
\end{array}\right] .
\end{aligned}
$$

\section{B. Minimum detectable fault}

In Section III, the formulation of minimum detectable fault is explored for each type of faults, separately. As discussed 
in previous subsection, the interval of the healthy residual invariant set can be computed off-line. This healthy residual set is considered for calculating the minimum detectable fault. In the following parts of the paper, the minimum detectable fault is computed based on the mathematical formulations presented in Section III.

1) Minimum detectable output sensor fault: As mentioned before, the minimum detectable output sensor fault is computed by (20) and (36). These expressions are used for the example application case. First approach for computing this magnitude is based on sensitivity and the second one is based on interval observer.

The influence of the gain and uncertainties on the magnitude can be seen on estimating the minimum detectable fault formulation. Therefore, gain, disturbance and noise change their magnitude during the simulation. Thus, the minimum detectable fault can be computed at each time step. The average of minimum magnitudes of the output sensor fault that can be detected is shown in Figure 1.

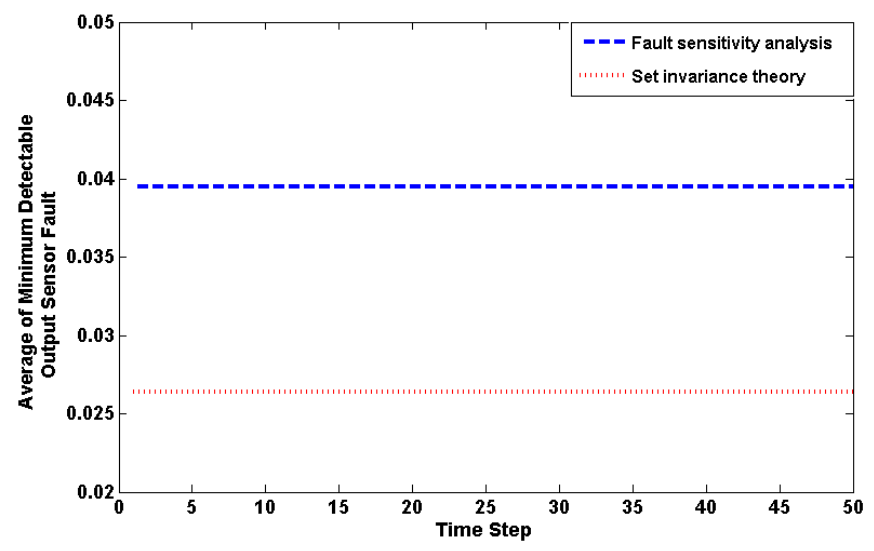

Fig. 1: The average of minimum detectable output sensor fault.

Dashed line shows the average of minimum detectable fault based on residual sensitivity and dotted line presents this value base on invariant set in Figure 1. From Figure 1, the average of minimum debatable output sensor fault that is obtained by the invariant set is smaller than the same value in sensitivity method. A possible explanation for this issue is to use the interval hull of the zonotope in comparison with the use of the exact zonotope. This fact has its influence on the residual sets that are created by interval observer and its invariant set.

2) Minimum Detectable Input Sensor Fault: Similar to the case of output sensor fault, the formulation of the minimum detectable input sensor fault is derived in (24) and (39) based on residual sensitivity and interval observer, respectively. The simulation is done with additive input sensor fault for the case study.

Therefore, the average of the minimum detectable fault during the simulation is shown in Figure 2.

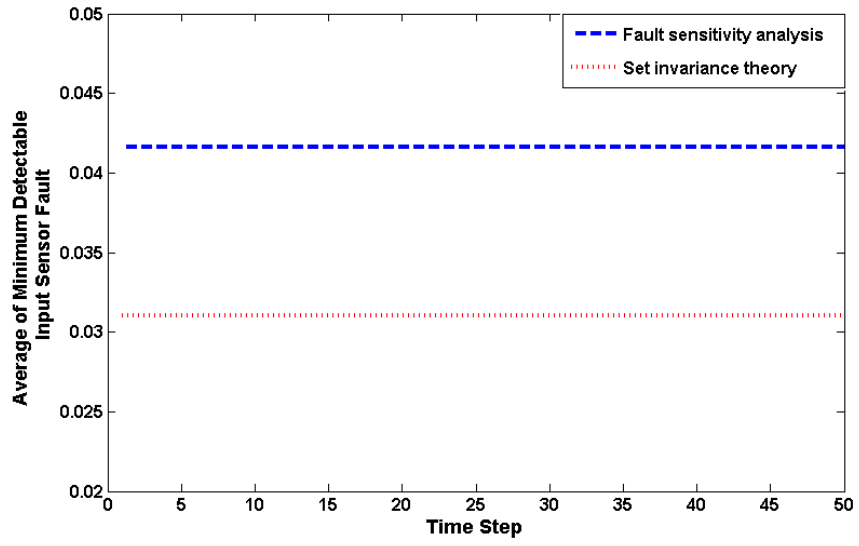

Fig. 2: The average of minimum detectable input sensor fault.

As it is seen in Figure 2, the dashed line shows the average of minimum detectable fault based on residual sensitivity and dotted line presents this value base on invariant set. Also, Figure 2 shows with interval-observer-based case the smaller magnitude of the detectable fault is computed during the simulation.

3) Minimum Detectable Actuator Fault: The other type of fault that is considered in this paper is the case of actuator fault. The minimum detectable fault is obtained by implementing (28) and (42).

In this case, the average of obtained values by each method are presented in Figure 3. Similarly, dashed line is used for showing the average of minimum detectable fault based on residual sensitivity and dotted line presents this value based on invariant sets in this case. As it can be observed, these magnitudes are different because of the influence of being over-approximated by means of the zonotope interval hull.

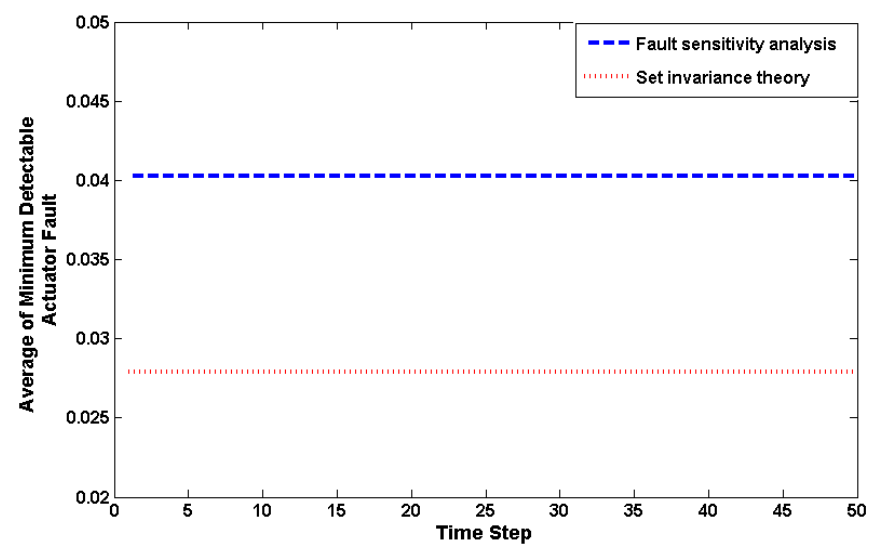

Fig. 3: The average of minimum detectable actuator fault.

\section{Fault Detection}

The case of actuator faults is used for illustrating the fault detection analysis presented in the paper. The actuator fault is injected as indicated in (25). Moreover, the actuator fault is added to the system at $k=20$ and it remains until time step $k=30$ in the all simulations.

Due to the occurrence of the actuator fault in the system, the residual set has been affected. Therefore, this fault is 
detected whenever the residual set goes into the set of faulty mode.

Additionally, three different cases can be considered for testing the fault detection performance based on the magnitude of the fault that are obtained using equations in (28) and (42) for sensitivity analysis and set invariance theory, respectively.

Firstly, a slightly bigger magnitude of the fault than the minimum detectable one that is obtained using (28) is applied.

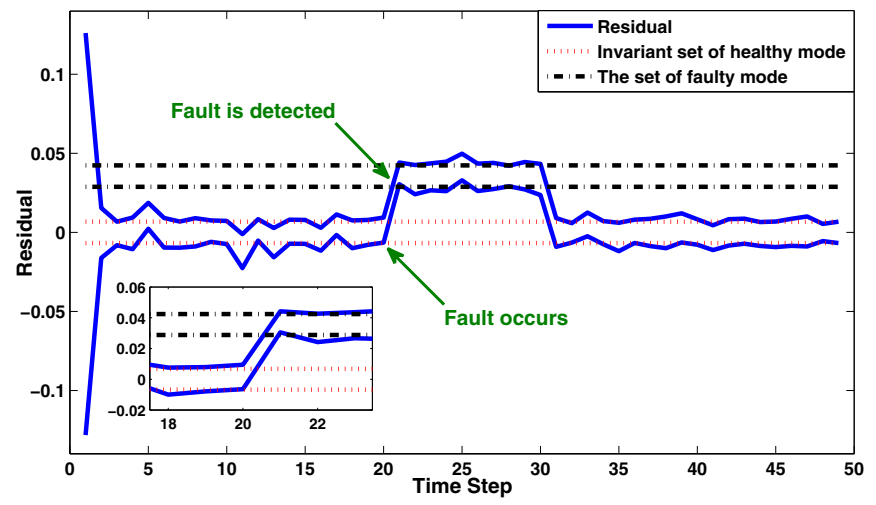

Fig. 4: Fault detection test of the system with occurrence of actuator fault with bigger magnitude of the fault than the minimum detectable one obtained using sensitivity analysis (Fault magnitude is bigger than $f_{a}^{\min }=0.04$ ).

Figure 4 shows the case that the actuator is healthy from time instants 0 to 20, from time instants 20 to 30 an actuator fault occurs and from time instants 30 to 50 the actuator recovers to the healthy mode. Also, the set of healthy and faulty modes can be seen in Figure 4 . From time step 0 to 20 , the actuator is healthy, thus, the residual zonotopes are contained by the healthy invariant set. At time step 20, the fault occurs, then the healthy invariant set excludes the residual zonotope. Furthermore, the fault is detected at time instant 21 , when the residual zonotope goes inside of the set of faulty mode.

The magnitude of the fault is considered bigger than the minimum detectable fault that is computed by equation in (28) with sensitivity analysis (fault magnitude is bigger than $f_{a}^{m i n}=0.04$ in Figure 4). Moreover, changing the residual set (from the healthy invariant set to the set of faulty mode) in Figure 4 pointing out that the occurrence of the actuator fault with this magnitude can be strongly detectable. Therefore, the fault will be detected if its magnitude is bigger than one that is computed by sensitivity analysis.

Secondly, a slightly smaller fault than the one established by (42) is applied.

The magnitude of the fault is considered smaller than $f_{a}^{m i n}=0.027$ in Figure 5. Also, Figure 5 shows that the fault occurs in actuator from $k=20$ to $k=30$ but the residual zonotope is still contained by healthy invariant set. Therefore, occurrence of the actuator fault with this magnitude will not be able to change the residual set from the healthy invariant set to the faulty mode set. Thus, the fault detection

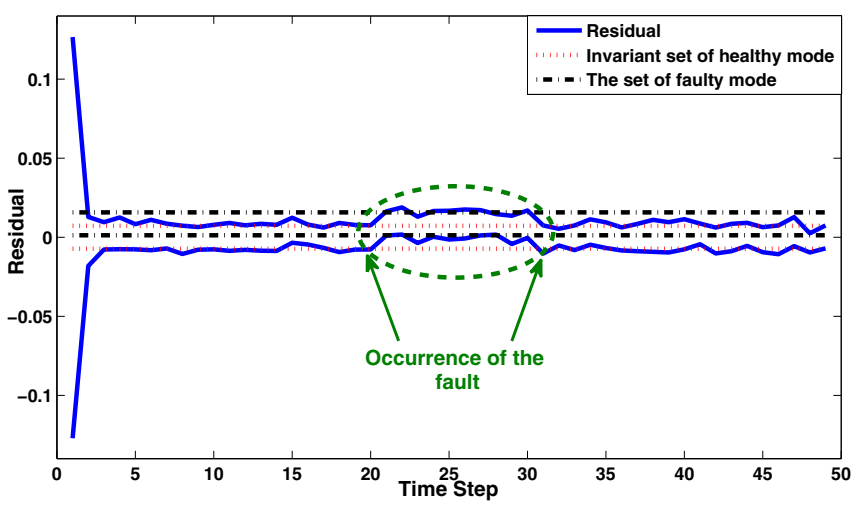

Fig. 5: Fault detection test of the system with occurrence of actuator fault with smaller magnitude of the fault than the minimum detectable one obtained using set-invariance theory (Fault magnitude is smaller than $f_{a}^{\min }=0.027$ ).

test is not capable of detecting the actuator fault with this magnitude. Moreover, the magnitudes of the actuator fault that are smaller than ones obtained by the set invariance theory are not detectable in this simulation.

Finally, the magnitude of the actuator fault is considered between one obtained using equation in (28) and one that is established by (42).

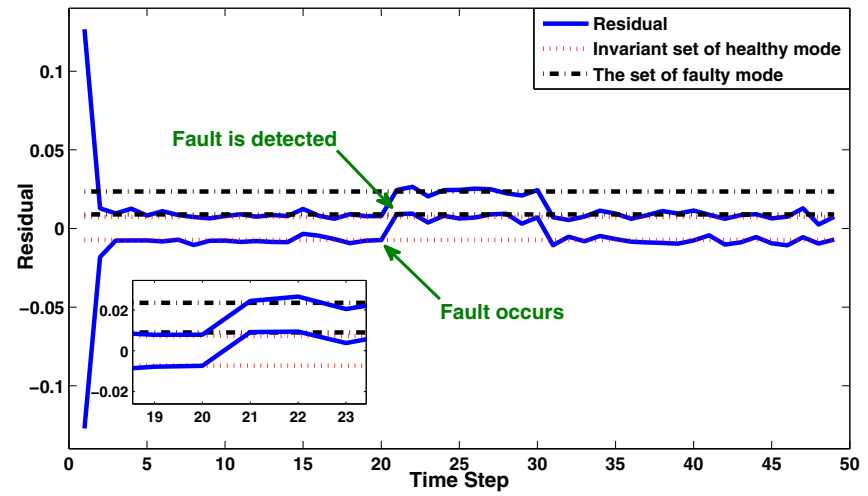

Fig. 6: Fault detection test of the system with occurrence of actuator fault with the magnitude between the minimum detectable fault that are obtained from sensitivity analysis and set-invariance theory $\left(0.027<f_{a}<0.04\right)$.

Figure 6 shows the influence of the fault on the residual when the magnitude of the fault is considered between the minimum detectable fault that are obtained from sensitivity analysis and set-invariance theory. Therefore, the magnitude of the actuator fault $f_{a}$ is considered between 0.027 and 0.04 $\left(0.027<f_{a}<0.04\right)$.

As it can be seen in Figure 6, at time step 20, the fault occurs, then the healthy invariant set excludes the residual zonotope. But due to the magnitude of the fault the set of faulty mode is quite close to the set of healthy mode. Therefore, if the residual zonotope goes inside of the faulty set based on uncertainties, it will be detected as a fault. Thus, the fault detection test can not be able to identify the 
influence of uncertainties on the residual (maybe the residual set is changed but this is not the effect of fault). Hence, the fault detection is known as weak fault detection and it is not reliable.

\section{Conclusions}

In this paper, the computation of minimum detectable fault has been studied for uncertain systems when the interval observer approach is used. This study has been carried out by using two approaches. First, sensitivity of the residual to the fault was considered. Second, the minimum detectable fault is computed by using set invariance theory. Comparing both approaches, it can be observed that the set invariance-based approach shows smaller value of the minimum detectable fault in comparison with sensitivity-based approach. In other words, the sensitivity-based approach is more conservative than the set invariance-based approach. These results have been established for the linear discrete-time case and the fault estimation problems for more complicated systems, like hybrid case, would be one of the future research topics.

\section{APPENDIX}

In what follows, some fundamental definitions and properties regarding zonotopes that are important to recall for understanding the contents of this paper, are introduced.

Definition 1 (Minkowski Sum): The Minkowski sum of two sets is defined by $X \oplus Y=\{x+y: x \in X, y \in Y\}$.

Definition 2 (m-order Zonotope): A zonotope is a convex symmetric polytope. Given a vector $p \in \mathbb{R}^{n}$ and a matrix $H \in \mathbb{R}^{n \times m}(n \leq m)$, the zonotope is represented as $Z=$ $p \oplus H \mathbb{B}^{m}=\left\{p+H z: z \in \mathbb{B}^{m}\right\}$, where $p$ is the center, $H$ contains the segments of the zonotope and $\mathbb{B}^{m}$ is an $m$ dimensional unitary box. Also, the order $m$ is a measure for the geometrical complexity of the zonotopes.

Definition 3 (Strip): A strip is described by $S=$ $\left\{x:\left|c^{T} x-d\right| \leq \sigma\right\}$, where $c$ is a vector and $d, \sigma$ are scalars.

Definition 4 (Interval Hull): Interval hull of a given zonotope $Z=p \oplus H \mathbb{B}^{m} \subset \mathbb{R}^{n}$ is the smallest interval box that contain $Z$ and it is denoted by $\square Z$.

Definition 5 (Invariant-set): The invariant-set $\Omega \subseteq X$ is the set which its existence allowed the evolution of a constrained system, where $x_{0} \in \Omega \subseteq X$ and then, $x_{k} \in \Omega \subseteq X$ for all time steps $k$.

Property 1: Given two zonotopes $Z_{1}=p_{1} \oplus H \mathbb{B}^{m_{1}} \in$ $\mathbb{R}^{n}$ and $Z_{2}=p_{1} \oplus H \mathbb{B}^{m_{2}} \in \mathbb{R}^{n}$, the Minkowski sum of these zonotopes is defined as $Z=Z_{1} \oplus Z_{2}=\left(p_{1}+p_{2} \oplus\right.$ $\left[\begin{array}{ll}H_{1} & H_{2}\end{array}\right] \mathbb{B}^{m_{1}+m_{2}}$, that is still a zonotope.

Property 2: Given the zonotope $Z=p \oplus H \mathbb{B}^{m} \subset \mathbb{R}^{n}$, the strip $S=\left\{x \in \mathbb{R}^{n}:\left|c^{T} x-d\right| \leq \sigma\right\}$ and the vector $\lambda \in \mathbb{R}^{n}$, the intersection between the zonotope and the strip is defined as $Z \cap S=\hat{p}(\lambda) \oplus \hat{H}(\lambda) \mathbb{B}^{m+1}$, where $\hat{p}(\lambda)=p+\lambda(d-c p)$ and $\hat{H}(\lambda)=\left[\begin{array}{ll}(I-\lambda c) H & \sigma \lambda\end{array}\right]$.

\section{ACKNOWLEDGMENT}

This work has been partially funded by the Spanish Ministry of Science and Technology through the Project ECOCIS (Ref. DPI2013-48243-C2-1-R) and Project HARCRICS (Ref. DPI2014-58104-R).

\section{REFERENCES}

[1] F. J. Bejarano, T. Floquet, W. Perruquetti, and G. Zheng. Observability and detectability analysis of singular linear systems with unknown inputs. In 50th IEEE Conference on Decision and Control and European Control Conference, pages 4005-4010, USA, 2011.

[2] C. Combastel. Zonotopes and kalman observers: Gain optimality under distinct uncertainty paradigms and robust convergence. Automatica, 55:265-273, 2015.

[3] H. Dong, Z. Wang, S. X. Ding, and H. Gao. Finite-horizon estimation of randomly occurring faults for a class of nonlinear time-varying systems. Automatica, 50(12):3182-3189, 2014.

[4] D. Efimov, A. Zolghadri, and T. Raïssi. Actuator fault detection and compensation under feedback control. Automatica, 47(8):1699-1705, 2011.

[5] H. Hamidi, A. Vafaei, and A. Monadjemi. A framework for fault tolerance techniques in the analysis and evaluation of computing systems. International journal of innovative computing, information and control, 8(7):5083-5094, 2012.

[6] K. H. Johansson. The quadruple-tank process: A multivariable laboratory process with an adjustable zero. IEEE Transactions on Control Systems Technology, 8(3):456-465, 2000.

[7] Q. Khan, A. I. Bhatti, M. Iqbal, and Q. Ahmed. Dynamic integral sliding mode control for SISO uncertain nonlinear systems. International Journal of Innovative Computing, Information and Control, 8(7):4621-4633, 2012.

[8] M. Liu, X. Cao, and P. Shi. Fuzzy-model-based fault-tolerant design for nonlinear stochastic systems against simultaneous sensor and actuator faults. IEEE Transactions on Fuzzy Systems, 21(5):789-799, 2013.

[9] J. Meseguer, V. Puig, T. Escobet, and J. Saludes. Observer gain effect in linear interval observer-based fault detection. Journal of Process Control, 20(8):944-956, 2010.

[10] C. Ocampo-Martinez, J. A. De Doná, and M. M. Seron. Actuator fault-tolerant control based on set separation. International Journal of Adaptive Control and Signal Processing, 24(12):1070-1090, 2010.

[11] B. Shen, S. X Ding, and Z. Wang. Finite-horizon $h_{\infty}$ fault estimation for linear discrete time-varying systems with delayed measurements. Automatica, 49(1):293-296, 2013.

[12] S. M. Tabatabaeipour and T. Bak. Robust observer-based fault estimation and accommodation of discrete-time piecewise linear systems. Journal of the Franklin Institute, 351(1):277-295, 2014.

[13] C. Tan, G. Tao, and R. Qi. A discrete-time parameter estimation based adaptive actuator failure compensation control scheme. International Journal of Control, 86(2):276-289, 2013.

[14] X. Wan, H. Fang, and S. Fu. Observer-based fault detection for networked discrete-time infinite-distributed delay systems with packet dropouts. Applied Mathematical Modelling, 36(1):270-278, 2012.

[15] F. Xu, V. Puig, C. Ocampo-Martinez, F. Stoican, and S. Olaru. Actuator-fault detection and isolation based on interval observers and invariant sets. In 52nd IEEE Annual Conference on Decision and Control, pages 4385-4390, Italy, 2013.

[16] F. Xu, F. Stoican, V. Puig, C. Ocampo-Martinez, and S. Olaru. On the relationship between interval observers and invariant sets in fault detection. In Conference on Control and Fault-Tolerant Systems, pages 49-54, France, 2013.

[17] K. Zhang, B. Jiang, V. Cocquempot, and H. Zhang. A framework of robust fault estimation observer design for continuous-time/discretetime systems. Optimal Control Applications and Methods, 34(4):442457, 2013.

[18] K. Zhang, B. Jiang, and P. Shi. Fast fault estimation and accommodation for dynamical systems. IET Control Theory \& Applications, 3(2):189-199, 2009.

[19] L. Zhang, E. K. Boukas, L. Baron, and H. R. Karimi. Fault detection for discrete-time Markov jump linear systems with partially known transition probabilities. International Journal of Control, 83(8):15641572,2010 . 